International Journal of Bifurcation and Chaos, Vol. 18, No. 11 (2008) 3341-3354

(c) World Scientific Publishing Company

\title{
SYNCHRONIZATION ERRORS AND UNIFORM SYNCHRONIZATION WITH AN ERROR BOUND FOR CHAOTIC SYSTEMS*
}

\author{
BIN LIU ${ }^{\dagger}$ \\ Department of Information and Computation Sciences, \\ Hunan University of Technology, \\ Zhuzhou 412008, P. R. China \\ bin.liu@anu.edu.au \\ DAVID J. HILL \\ ${ }^{\dagger}$ Department of Information Engineering, \\ Australian National University, \\ ACT 0200, Australia \\ GUANRONG CHEN \\ Department of Electronic Engineering, \\ City University of Hong Kong, \\ Kowloon, Hong Kong, P. R. China
}

Received October 22, 2007; Revised December 3, 2007

\begin{abstract}
This paper investigates the problem of estimating synchronization errors and its application to uniform synchronization with an error bound for the general master-slave chaos synchronization scheme via feedback control, which is subjected to disturbances by unknown but bounded channel noise. Based on the Lyapunov function and nonlinear parametric variation techniques, estimation formulae for synchronization errors are derived. It is possible to synchronize two master-slave chaotic systems with a relatively small error bound, even in the case with unknown but bounded noisy disturbances. After the theoretical analysis, some representative examples and their numerical simulations are given for illustration.
\end{abstract}

Keywords: Chaos synchronization; error bound; synchronization error; exponential synchronization; uniform synchronization; variation of parameters.

\section{Introduction}

Chaos synchronization has attracted increasing attention due to its great potential in some engineering applications such as biological systems (neural network functioning, brain activities, heartbeat regulation, etc.), oscillators design, vibrating wave generation, mechanical resonance, spatiotemporal pattern formation and secure communications. Many strategies and methods for chaos synchronization have been developed since the early 1990s [Pecora \& Carroll, 1990; Carroll \& Pecora, 1991; Kocarev \& Parlitz, 1995; Wu et al., 1996; Yang \& Chua, 1997; Grassi \& Mascolo, 1997; Fang \& Hong, 1999; Kocarev et al., 2001], among

\footnotetext{
*This work was supported by the ARC-Australia (No. FF0455875 and No. DP0881391) and NSFC-China (No. 60874025).

${ }^{\dagger}$ Author for correspondence
} 
which feedback control is especially attractive and effective. Many results on chaos synchronization via feedback control are now available in the literature [Chen \& Dong, 1998; Chen \& Lai, 1996; Chen, 2000; Yalcin et al., 2001; Wu \& Chua, 1994]. More recently, synchronization of complex dynamical networks has also received much attention, see, for example, [Pecora et al., 2000; Wu, 2003; Wang \& Chen, 2002; Li \& Chen, 2004b; Liu et al., 2005; Rodrigues et al., 2000; Yang, 2001; Lü \& Chen, 2005; Jiang et al., 2006; Xiang \& Chen, 2007].

However, it has been observed that when a synchronization scheme is applied to chaotic systems or a dynamical network in engineering applications like communications, there are many factors which may cause the failure of the synchronization scheme, particularly, (i) their susceptibility to noise and distortions; and (ii) time delays through transmission channels. Channel noise may be presented in different forms, and usually destroys good properties of synchronized systems. Moreover, noise in many real chaotic synchronization systems are difficult to know beforehand except their bounds. Time delays, on the other hand, occur commonly in synchronization schemes. To resolve these problems, a robust synchronization theory has been developed and is very promising. References [Liu et al., 2007; Liu \& Marquez, 2008; Li \& Chen, 2004a] study synchronization of time-delay dynamical networks. Meanwhile, robust synchronization in the case of parameter mismatch in nonidentical Lur'e chaotic systems and dynamical networks have been investigated [Curran \& Chua, 1997; Suykens et al., 1997; Suykens et al., 1999; Pecora et al., 2000; Wu, 2003; Wang \& Chen, 2002; Li \& Chen, 2004b; Liu et al., 2005], but there are very few theoretical results for general synchronization in which the channel is disturbed by unknown noise. To our knowledge, no investigation has been carried out for the estimation of synchronization errors subject to unknown channel noise to date.

In this paper, a chaos synchronization problem subject to noise disturbances is considered for general chaotic systems. More precisely, the estimation problem of synchronization errors is studied, where the channel is subjected to unknown but bounded noise disturbances, for a setting of general master-slave chaos synchronization. By employing the methods of Lyapunov functions and nonlinear variation of parameters, an estimation formula for synchronization errors will be derived. By using the derived estimation formula, one can design a feedback controller using which it is possible to synchronize master-slave chaotic systems up to a relatively small error bound, even in the case of unknown but bounded noise disturbances. Moreover, some exponential synchronization criteria will be derived for the noise-free situation. Simulations will be given to verify the theoretical results.

The rest of this paper is organized as follows. In Sec. 2, the master-slave synchronization scheme for general chaotic systems is first formulated, subject to unknown but bounded noise disturbances. The approach to achieving synchronization via feedback control is also proposed in this section. Then, in Sec. 3, several estimation formulae for synchronization errors and exponential synchronization criteria are established. These results can be easily used for the design of the feedback controller. In Sec. 4, some representative examples are given for illustration.

\section{Preliminaries}

In the sequel, $R$ denotes the field of real number, $R^{+}$ the subset of non-negative elements of $R$, defined by $R^{+}=[0,+\infty)$, and $R^{n}$ the $n$-dimensional Euclidean space. Given a matrix $A,\|A\|$ denote the norm of $A$ induced by the Euclidean vector norm, i.e. $\|A\|=\left[\lambda_{\max }\left(A^{T} A\right)\right]^{\frac{1}{2}}$. A function $\gamma: R^{+} \rightarrow R^{+}$ is of class- $\mathcal{K}(\gamma \in \mathcal{K})$ if it is continuous, zero at zero and strictly increasing. A continuous function $\beta: R_{+} \times R_{+} \rightarrow R_{+}$is of class- $\mathcal{K} \mathcal{L}$ if $\beta(\cdot, t)$ is of class- $\mathcal{K}$ for each $t \geq 0$ and $\beta(s, \cdot)$ is monotonically decreasing to zero for each $s>0$.

Consider the chaos synchronization scheme via feedback control:

The master system:

$$
M:\left\{\begin{array}{l}
\dot{x}(t)=f(t, x(t)), \\
p(t)=H x(t),
\end{array}\right.
$$

where $x, p \in R^{n}, f \in C\left[R_{+} \times R^{n}, R^{n}\right]$, and $H \in$ $R^{n \times n}$ is a nonsingular matrix.

The slave system:

$$
S:\left\{\begin{array}{l}
\dot{y}(t)=f(t, y(t))+u(t), \\
q(t)=H y(t) .
\end{array}\right.
$$

The feedback controller is designed as

$$
C: u(t)=F(p(t)-q(t)),
$$

where $F \in R^{n \times n}$ is a constant control gain matrix.

If the transmission channel is not contaminated with noise, the synchronization error is defined as

$$
e(t)=x(t)-y(t)
$$


Thus, one has an error dynamical system of the form

$$
\dot{e}(t)=f(t, x(t))-f(t, y(t))-F H e(t) .
$$

Denote $g(t, e, y)=f(t, e+y)-f(t, y)-F H e$. Then, (4) can be rewritten as

$$
\dot{e}=g(t, e, y) .
$$

In applications, when the signals are transmitted from the master system to the slave system, the transmission channel is unavoidably contaminated with noise. Denote the noise by $\omega(t)$ at time $t$, which is assumed to be unknown but bounded. Figure 1 depicts the entire setting with noise disturbances.

Thus, the real control signal being received and used by the slave system at time $t$ is

$$
\begin{aligned}
v(t) & =F[(p(t)-q(t))+\omega(t)] \\
& =F H(x(t)-y(t))+F \omega(t) .
\end{aligned}
$$

In this case, the slave system becomes:

$$
\tilde{S}:\left\{\begin{array}{l}
\dot{\tilde{y}}(t)=f(t, \tilde{y}(t))+v(t), \\
\tilde{q}(t)=H \tilde{y}(t) .
\end{array}\right.
$$

Define the new synchronization error as

$$
r(t)=x(t)-\tilde{y}(t) .
$$

Then, one has the actual error dynamical system of the form

$$
\dot{r}(t)=g(t, r, \tilde{y})-F \omega(t) .
$$

Definition 2.1. The noise-free synchronization scheme (1)-(3) is said to achieve exponential synchronization if, for any initial condition $x_{0}$, the trivial solution $e=0$ of the error dynamical system (5) is exponentially stable, in the sense that there exist

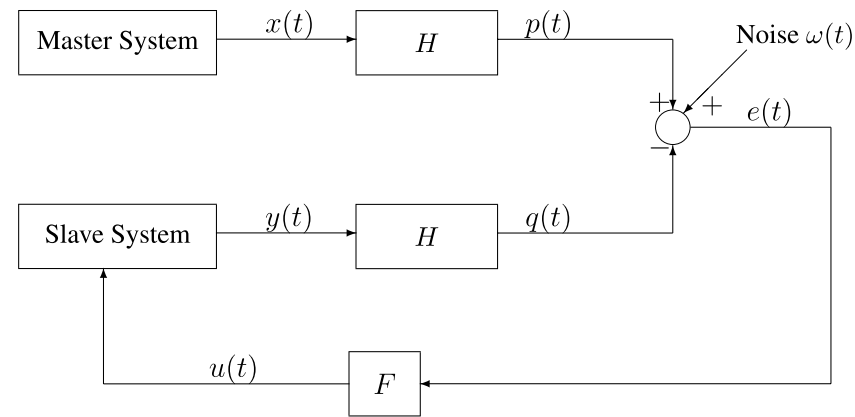

Fig. 1. The chaos synchronization framework subject to noise disturbances. two positive numbers, $\alpha>0, M \geq 1$, such that

$$
\begin{aligned}
\|e(t)\| & =\|x(t)-y(t)\| \\
& \leq M\left\|x_{0}-y_{0}\right\| e^{-\alpha\left(t-t_{0}\right)}, \quad t \geq t_{0} .
\end{aligned}
$$

Definition 2.2. The actual synchronization scheme (1) with (7)-(8) is said to achieve uniform synchronization with an error bound $\epsilon$ if there exist a $\delta_{0}>0$ and a $T \geq t_{0}$ such that $\left\|r\left(t_{0}\right)\right\| \leq \delta_{0}$, implies $\|r(t)\| \leq \epsilon$ for all $t \geq T$.

Assumption 2.1. The noise may be unknown but satisfies $\omega(0)=0$ and $\|\omega(\cdot)\| \leq K$ for some positive constant $K>0$.

Assumption 2.2. [Curran \& Chua, 1997] There exists a positive real constant $\delta>0$ such that for any initial condition $\left(t_{0}, x_{0}\right) \in R^{+} \times R^{n}$ there exists a time $T\left(t_{0}, x_{0}\right) \geq t_{0}$ satisfying

$$
x\left(t, t_{0}, x_{0}\right) \in D \triangleq\{x \mid\|x(t)\| \leq \delta, t \geq T\} .
$$

Assumption 2.3. $f$ satisfies the Lipschitz condition uniformly with respect to the second variable, namely, for some $L>0$,

$$
\begin{array}{r}
\|f(t, x)-f(t, y)\| \leq L\|x-y\|, \\
\text { for all } t \geq t_{0}, \quad x, y \in D .
\end{array}
$$

The main objective of this paper is to estimate the bound $\epsilon$ of the synchronization error $\|r(t)\|$, as specified in Definition 2.2, when the transmission channel is contaminated with unknown but bounded noise. To do so, we need the following preliminaries on nonlinear variation of parameters.

Consider the following nonlinear system:

$$
\dot{z}=h(t, z),
$$

and its perturbed system:

$$
\dot{\tilde{z}}=h(t, \tilde{z})+\varphi(t, \tilde{z}),
$$

where $h$ is continuously differentiable in region $\left[t_{0}, \infty\right) \times \tilde{D}$, with $\tilde{D} \subseteq R^{n}$, and $\varphi$ is continuous in $\left[t_{0}, \infty\right) \times \tilde{D}$. Let $z\left(t, t_{0}, z_{0}\right)$ denote the solution of the nonlinear system (12) passing through the initial point $\left(t_{0}, z_{0}\right)$, and let $\tilde{z}\left(t, t_{0}, \tilde{z}_{0}\right)$ be that of (13).

Lemma 2.1 [Brauer, 1966]. If there exists a continuous function $\alpha$ on $\left[t_{0}, \infty\right)$ such that

$$
\begin{array}{r}
\frac{1}{2} \lambda_{\max }\left(h_{z}(t, z)+h_{z}^{T}(t, z)\right) \leq \alpha(t), \\
\forall t \geq t_{0}, \quad z \in \tilde{D},
\end{array}
$$


where $h_{z}(t, z)=\partial h(t, z) / \partial z$, and if $z_{0}, z_{1}$ are in a convex subset $\hat{D}$ of $\tilde{D}$, then for all $t$, for which all solutions with initial values in $\hat{D}$ at time $t_{0}$ will remain in $\tilde{D}$, one has

$$
\left\|z\left(t, t_{0}, z_{1}\right)-z\left(t, t_{0}, z_{0}\right)\right\| \leq\left\|z_{1}-z_{0}\right\| e^{\int_{t_{0}}^{t} \alpha(u) d u} .
$$

Lemma 2.2 [Brauer, 1966]. If (14) is satisfied and if $z_{0} \in \hat{D}, \tilde{z}_{0} \in \hat{D}$, then for all $t$, for which all solutions of (12) starting from $\hat{D}$ at time $t_{0}$ will remain in $\tilde{D}$ and similarly $\tilde{z}\left(t, t_{0}, \tilde{z}_{0}\right) \in \tilde{D}$, one has

$$
\begin{aligned}
& \left\|\tilde{z}\left(t, t_{0}, \tilde{z}_{0}\right)-z\left(t, t_{0}, z_{0}\right)\right\| \\
& \quad \leq\left\|\tilde{z}_{0}-z_{0}\right\| e^{\int_{t_{0}}^{t} \alpha(u) d u}+\int_{t_{0}}^{t} e^{\int_{s}^{t} \alpha(u) d u}\|\varphi(s)\| d s .
\end{aligned}
$$

Lemma 2.3 [Teel, 1998]. Let $\nu>0$ and $\alpha \in \mathcal{K}$. If $m(t) \geq \nu$ implies that $D^{+} m(t) \leq-\alpha(m(t))$, then there exists $\beta \in \mathcal{K} \mathcal{L}$ (independent of $\nu$ ) with $\beta(s, 0) \geq s$ such that

$$
m(t) \leq \max \left\{\beta\left(m\left(t_{0}\right), t-t_{0}\right), \nu\right\} .
$$

\section{Synchronization Error Estimations}

In this section, the problem of synchronization error estimation is studied. Some synchronization error formulae are derived under which uniform synchronization can be achieved with an error bound.

Comparing (8) with (5), one can rewrite system (8) as follows:

$$
\dot{r}=g(t, r, y)+W(t),
$$

where $W(t)=g(t, r(t), \tilde{y}(t))-g(t, r(t), y(t))-$ $F \omega(t)$. From (4), it is easy to see that

$$
\begin{aligned}
W(t)= & {[f(t, r(t)+\tilde{y}(t))-f(t, r(t)+y(t))] } \\
& +[f(t, y(t))-f(t, \tilde{y}(t))]-F \omega(t),
\end{aligned}
$$

and by Assumption 2.1, $W(t)$ satisfies

$$
\begin{array}{r}
\|W(t)\| \leq 2 L\|\tilde{y}(t)-y(t)\|+K\|F\|, \\
\text { for all } t \geq t_{0} .
\end{array}
$$

Theorem 3.1. Let Assumptions 2.1-2.3 hold and also assume that there exist a control gain matrix $F$, a positive definite matrix $P>0$, and a positive constant $p(F, P)$ with $p(F, P)>2 L \lambda_{\max }(P) \lambda_{\min }(P)$ such that for any solution $r$ of (18),

$$
r^{T} P g(t, r, y) \leq-p(F, P) r^{T} P r, \quad \forall t \geq t_{0}, \quad y \in D .
$$

Then, the noise-free synchronization scheme (1)(3) is exponentially synchronizing and the actual synchronization scheme (1) with (7)-(8) is uniformly synchronizing with an error bound

$$
\left\{\frac{\lambda_{\max }(P)}{\lambda_{\min }(P)}\right\}^{\frac{1}{2}} \frac{K\|P F\| \lambda_{\min }(P)}{\left(p(F, P)-2 L \lambda_{\max }(P) \lambda_{\min }(P)\right)} .
$$

Proof. Let $V(\xi)=\xi^{T} P \xi$, with $\xi \in R^{n}$. Let $e\left(t, t_{0}, e_{0}\right)$ denote the solution of (5) passing through the initial point $\left(t_{0}, e_{0}\right)$, and let $r\left(t, t_{0}, r_{0}\right)$ be the solution of (18) passing through the initial point $\left(t_{0}, r_{0}\right)$. By $(20)$, one has

$$
\begin{aligned}
\left.D^{+} V(e(t))\right|_{(5)} & =2 e^{T} P g(t, e, y) \\
& \leq-2 p(F, P) V(e(t)) .
\end{aligned}
$$

Thus, for all $t \geq t_{0}$, one has $V(e(t)) \leq$ $V\left(e_{0}\right) e^{-2 p(F, P)\left(t-t_{0}\right)}$, which implies that

$$
\|e(t)\| \leq \sqrt{\frac{\lambda_{\max }(P)}{\lambda_{\min }(P)}}\left\|e_{0}\right\| e^{-p(F, P)\left(t-t_{0}\right)} .
$$

Therefore, the scheme (1)-(3) is exponentially synchronizing.

Now, examine the actual synchronization error $\|r(t)\|$. It follows from (20) that

$$
\begin{aligned}
D^{+} V( & r(t))\left.\right|_{(18)} \\
= & 2 r^{T} P g(t, r, y)+2 r^{T} P W(t) \\
\leq & -2 p(F, P) r^{T} P r+2 r^{T} P\{[f(t, r+\tilde{y}) \\
& -f(t, r+y)]+[f(t, y)-f(t, \tilde{y})]-F \omega(t)\} \\
\leq & -2 p(F, P) V(r)+4 L\|r\|\|P\|\|\tilde{y}-y\| \\
& -2 r^{T} P F \omega(t) \\
= & -\frac{p(F, P)}{\lambda_{\min }(P)}\|r\|^{2}+4 L\|P\|\|r\|\|r-e\| \\
& +2 K\|P F\|\|r\| \\
\leq & 2\|r\|\left(-\left(\frac{2 p(F, P)}{\lambda_{\min }(P)}-2 L\|P\|\right)\|r\|\right. \\
& +2 L\|P\|\|e\|+K\|P F\|) .
\end{aligned}
$$

By Assumption 2.2 and (22)-(23), we get that for any $T^{\prime} \geq T\left(t_{0}, x_{0}\right)$, and $t \geq T^{\prime}$,

$$
\begin{aligned}
\left.D^{+} V(r(t))\right|_{(18)} & \\
\leq & 2\|r\|\left(-\left(\frac{p(F, P)}{\lambda_{\min }(P)}-2 L\|P\|\right)\|r\|\right. \\
& \left.+2 L\|P\|\left\|e_{0}\right\| e^{-p(F, P)\left(T^{\prime}-t_{0}\right)}+K\|P F\|\right) .
\end{aligned}
$$


Thus, for fixed $T^{\prime}$ and any $\epsilon>0$, from (24), if

$$
\|r(t)\| \geq \frac{K\|P F\|}{\frac{p(F, P)}{\lambda_{\min }(P)}-2 L\|P\|}+\frac{2 L\|P\|}{\frac{p(F, P)}{\lambda_{\min }(P)}-2 L\|P\|}\left\|e_{0}\right\| e^{-p(F, P)\left(T^{\prime}-t_{0}\right)}+\epsilon,
$$

then

$$
\left.D^{+} V(r(t))\right|_{(18)} \leq-2 \epsilon\left(\frac{p(F, P)}{\lambda_{\min }(P)}-2 L\|P\|\right)\|r\|, \quad \text { for all } t \geq T^{\prime}
$$

Hence, if

$$
V(r) \geq \lambda_{\max }(P)\left\{\frac{K\|P F\|}{\frac{p(F, P)}{\lambda_{\min }(P)}-2 L\|P\|}+\frac{2 L\|P\|}{\frac{p(F, P)}{\lambda_{\min }(P)}-2 L\|P\|}\left\|e_{0}\right\| e^{-p(F, P)\left(T^{\prime}-t_{0}\right)}+\epsilon\right\}^{2},
$$

then (25) holds. Thus, let $\alpha(s)=2 \epsilon\left(\left(p(F, P) / \lambda_{\min }(P)\right)-2 L\|P\|\right)\left(1 / \sqrt{\lambda_{\max }(P)}\right) \sqrt{s}$ for any $s \in R^{+}$, by Lemma 2.3 , there exists $\beta \in \mathcal{K} \mathcal{L}$ such that for any $t \geq T^{\prime}$,

$V(r) \leq \beta\left(\left\|V\left(T^{\prime}\right)\right\|, t-T^{\prime}\right)+\lambda_{\max }(P)\left\{\frac{K\|P F\|}{\frac{p(F, P)}{\lambda_{\min }(P)}-2 L\|P\|}+\frac{2 L\|P\|}{\frac{p(F, P)}{\lambda_{\min }(P)}-2 L\|P\|}\left\|e_{0}\right\| e^{-p(F, P)\left(T^{\prime}-t_{0}\right)}+\epsilon\right\}^{2}$.

Thus, for any $t \geq T^{\prime}$, it yields that the estimation of $\|r(t)\|$ is

$$
\begin{aligned}
\|r(t)\| \leq & \left\{\frac{\beta\left(\left\|V\left(T^{\prime}\right)\right\|, t-T^{\prime}\right)}{\lambda_{\min }(P)}+\frac{\lambda_{\max }(P)}{\lambda_{\min }(P)}\left\{\frac{K\|P F\|}{\frac{p(F, P)}{\lambda_{\min }(P)}-2 L\|P\|}\right.\right. \\
& \left.\left.+\frac{2 L\|P\|}{\frac{p(F, P)}{\lambda_{\min }(P)}-2 L\|P\|}\left\|e_{0}\right\| e^{-p(F, P)\left(T^{\prime}-t_{0}\right)}+\epsilon\right\}\right\}^{2}, \quad t \geq T^{\prime} .
\end{aligned}
$$

It yields that

$$
\lim _{t \rightarrow \infty} \sup \|r(t)\| \leq\left\{\frac{\lambda_{\max }(P)}{\lambda_{\min }(P)}\left\{\frac{K\|P F\|}{\frac{p(F, P)}{\lambda_{\min }(P)}-2 L\|P\|}+\frac{2 L\|P\|}{\frac{p(F, P)}{\lambda_{\min }(P)}-2 L\|P\|}\left\|e_{0}\right\| e^{-p(F, P)\left(T^{\prime}-t_{0}\right)}+\epsilon\right\}^{2}\right\}^{\frac{1}{2}} .
$$

Thus, letting $T^{\prime} \rightarrow \infty$, and $\epsilon \rightarrow 0^{+}$, then,

$$
\begin{aligned}
\lim _{t \rightarrow \infty} \sup \|r(t)\| & \leq\left\{\frac{\lambda_{\max }(P)}{\lambda_{\min }(P)}\right\}^{\frac{1}{2}} \frac{K\|P F\| \lambda_{\min }(P)}{p(F, P)-2 L\|P\| \lambda_{\min }(P)} \\
& =\left\{\frac{\lambda_{\max }(P)}{\lambda_{\min }(P)}\right\}^{\frac{1}{2}} \frac{K\|P F\| \lambda_{\min }(P)}{p(F, P)-2 L \lambda_{\max }(P) \lambda_{\min }(P)} .
\end{aligned}
$$


Hence, the actual synchronization scheme (1) with (7)-(8) is uniformly synchronizing with an error bound

$$
\left\{\frac{\lambda_{\max }(P)}{\lambda_{\min }(P)}\right\}^{\frac{1}{2}} \frac{K\|P F\| \lambda_{\min }(P)}{p(F, P)-2 L \lambda_{\max }(P) \lambda_{\min }(P)} .
$$

The proof is thus complete.

Remark 3.1. Most chaotic systems are in the form of a Lur'e system: $f(x)=A x+\varphi(x)$, and there are many papers in the literature in which the feedback controller was designed and the condition (20) is necessary to achieve the synchronization. Hence, with the gain matrix $F$ and the positive definite matrix $P>0$, condition (20) is rational.

In the following, consider the case in which $f$ is a differentiable function. All notions are as above.

Theorem 3.2. Let Assumptions 2.1-2.3 hold and also assume that there exists a control gain matrix $F$ such that the following conditions hold:

(i) There exists a continuous function $\alpha_{F}$ on $t \geq t_{0}$ and a constant $T_{0}$ with $T_{0} \geq T$ such that

$$
\begin{aligned}
\frac{1}{2} \lambda_{\max } & \left\{(-F H)^{T}+(-F H)+f_{z}(t, z)\right. \\
& \left.+f_{z}(t, z)^{T}\right\} \\
\leq & \alpha_{F}(t) \leq 0, \quad \forall t \geq T_{0}, \quad z \in D ;
\end{aligned}
$$

(ii)

$$
\sigma(F) \triangleq \lim _{t \rightarrow \infty} \sup \frac{1}{t-t_{0}} \int_{t_{0}}^{t} \alpha_{F}(s) d s<0 .
$$

Then, the noise-free synchronization scheme (1)-(3) is exponentially synchronizing and the actual synchronization scheme (1) with (7)-(8) is uniformly synchronizing with an error bound $(2\|F\| K /|\sigma(F)|) e^{\frac{4 L}{\sigma(F) \mid}}$.

Proof. First, let us show that $\sigma(F) \neq-\infty$ for some suitable constant control gain matrix $F$.
Suppose that for some constant control gain matrix $F, \sigma(F)=-\infty$ holds. Then, one can change $F$ into $\tilde{F}=c H^{-1}+F$, with $c>0$, such that

$$
\begin{aligned}
\frac{1}{2} \lambda_{\max } & \left\{(-\tilde{F} H)^{T}+(-\tilde{F} H)+f_{z}(t, z)+f_{z}(t, z)^{T}\right\} \\
= & -c+\frac{1}{2} \lambda_{\max }\left\{(-F H)^{T}+(-F H)\right. \\
& \left.+f_{z}(t, z)+f_{z}(t, z)^{T}\right\} \\
\leq & -c+\alpha(t) \leq-c, \quad \forall t \geq T_{0} .
\end{aligned}
$$

In this case, let $\tilde{\alpha}_{\tilde{F}}(t)=-c$, and hence the condition (ii) implies $-\infty<\sigma(\tilde{F})=-c<0$.

Next, let us show that the noise-free synchronization scheme (1)-(3) is exponentially synchronizing.

Let $e\left(t, t_{0}, e_{0}\right)$ denote the solution of (5) passing through the initial point $\left(t_{0}, e_{0}\right)$, and let $r\left(t, t_{0}, r_{0}\right)$ be the solution of (18) passing through the initial point $\left(t_{0}, r_{0}\right)$. Obviously, $g(t, 0, y) \equiv 0$ for $t \geq t_{0}$, so $e=0$ is a solution of (5).

The condition (ii) implies that, for all sufficiently large $t$,

$$
\int_{t_{0}}^{t} \alpha_{F}(s) d s \leq \frac{\sigma(F)}{2}\left(t-t_{0}\right) .
$$

By Lemma 2.1, for sufficiently large $t$,

$$
\begin{aligned}
\left\|e\left(t, t_{0}, e_{0}\right)\right\| & \leq\left\|e_{0}\right\| e^{\int_{t_{0}}^{t} \alpha_{F}(u) d u} \\
& \leq\left\|e_{0}\right\| e^{\frac{\sigma(F)}{2}\left(t-t_{0}\right)} .
\end{aligned}
$$

This implies that the noise-free scheme $(1)-(3)$ is exponentially synchronizing.

Finally, let us estimate the synchronization error $\left\|r\left(t, t_{0}, r_{0}\right)\right\|$.

Comparing the error system (5) with its perturbed system (18), by Lemma 2.2, one has

$$
\begin{aligned}
&\left\|r\left(t, t_{0}, r_{0}\right)-e\left(t, t_{0}, e_{0}\right)\right\| \\
& \leq\left\|r_{0}-e_{0}\right\| e^{\int_{t_{0}}^{t} \alpha_{F}(u) d u} \\
&+\int_{t_{0}}^{t} e^{\int_{s}^{t} \alpha_{F}(u) d u}\|W(s)\| d s .
\end{aligned}
$$

Denote $\theta(t)=r\left(t, t_{0}, r_{0}\right)-e\left(t, t_{0}, e_{0}\right)$. It follows from (19) and (32) that

$$
\begin{aligned}
\|\theta(t)\| & =\left\|r\left(t, t_{0}, r_{0}\right)-e\left(t, t_{0}, e_{0}\right)\right\| \\
& \leq\left\|\theta_{0}\right\| e^{\frac{\sigma(F)}{2}\left(t-t_{0}\right)}+\int_{t_{0}}^{t} e^{\int_{s}^{t} \alpha_{F}(u) d u}\|W(s)\| d s \\
& \leq\left\|\theta_{0}\right\| e^{\frac{\sigma(F)}{2}\left(t-t_{0}\right)}+\int_{t_{0}}^{t} e^{\int_{s}^{t} \alpha_{F}(u) d u}[\|F\| K+2 L\|\theta(s)\|] d s
\end{aligned}
$$




$$
\begin{aligned}
& =\left\|\theta_{0}\right\| e^{\frac{\sigma(F)}{2}\left(t-t_{0}\right)}+\|F\| K \int_{t_{0}}^{t} e^{\int_{s}^{t} \alpha_{F}(u) d u} d s+2 L \int_{t_{0}}^{t} e^{\int_{s}^{t} \alpha_{F}(u) d u}\|\theta(s)\| d s \\
& \leq\left\|\theta_{0}\right\| e^{\frac{\sigma(F)}{2}\left(t-t_{0}\right)}+\|F\| K \int_{t_{0}}^{t} e^{\frac{\sigma(F)}{2}(t-s)} d s+2 L \int_{t_{0}}^{t} e^{\int_{s}^{t} \alpha_{F}(u) d u}\|\theta(s)\| d s \\
& =\frac{2\|F\| K}{|\sigma(F)|}+\left(\left\|\theta_{0}\right\|-\frac{2\|F\| K}{|\sigma(F)|}\right) e^{\frac{\sigma(F)}{2}\left(t-t_{0}\right)}+2 L \int_{t_{0}}^{t} e^{\int_{s}^{t} \alpha_{F}(u) d u}\|\theta(s)\| d s .
\end{aligned}
$$

Since $\sigma(F)<0$, for any sufficiently small $\varepsilon>0$, there exists a $T_{1} \geq T_{0}$ such that for $t \geq T_{1}$,

$$
e^{\frac{\sigma(F)}{2}\left(t-t_{0}\right)}<\varepsilon \text {. }
$$

By (35)-(36), for $t \geq T_{1}$, one has

$$
\begin{aligned}
\|\theta(t)\| \leq & \left(\frac{2\|F\| K}{|\sigma(F)|}+\varepsilon \cdot\left|\left\|\theta_{0}\right\|-\frac{2\|F\| K}{|\sigma(F)|}\right|\right) \\
& +2 L \int_{t_{0}}^{t} e^{\int_{s}^{t} \alpha_{F}(u) d u}\|\theta(s)\| d s .
\end{aligned}
$$

By the Gronwall-Bellman inequality, for $t \geq T_{1}$,

$$
\begin{aligned}
\|\theta(t)\| \leq & \left(\frac{2\|F\| K}{|\sigma(F)|}+\varepsilon \cdot\left|\left\|\theta_{0}\right\|-\frac{2\|F\| K}{|\sigma(F)|}\right|\right) \\
& \times e^{2 L \int_{t_{0}}^{t} e^{\int_{s}^{t} \alpha_{F}(u) d u} d s} \\
\leq & \left(\frac{2\|F\| K}{|\sigma(F)|}+\varepsilon \cdot\left|\left\|\theta_{0}\right\|-\frac{2\|F\| K}{|\sigma(F)|}\right|\right) \\
& \times e^{2 L\left[\frac{2}{|\sigma(F)|}\left(1-e^{\frac{\sigma(F)}{2}\left(t-t_{0}\right)}\right)\right] .}
\end{aligned}
$$

Thus,

$$
\begin{aligned}
\lim _{t \rightarrow \infty} \sup \|\theta(t)\| \leq & \left(\frac{2\|F\| K}{|\sigma(F)|}+\varepsilon \cdot\left|\left\|\theta_{0}\right\|-\frac{2\|F\| K}{|\sigma(F)|}\right|\right) \\
& \times e^{\frac{4 L}{\sigma(F)} .}
\end{aligned}
$$

Letting $\varepsilon \rightarrow 0$ yields

$$
\lim _{t \rightarrow \infty} \sup \|\theta(t)\| \leq \frac{2\|F\| K}{|\sigma(F)|} e^{\frac{4 L}{\sigma(F)}} .
$$

The exponential synchronization of scheme (1)-(3) implies that $\lim _{t \rightarrow \infty} e\left(t, t_{0}, e_{0}\right)=0$. Hence, from (40), one has

$$
\begin{aligned}
& \lim _{t \rightarrow \infty} \sup \left\|r\left(t, t_{0}, r_{0}\right)\right\| \\
& \quad \leq \lim _{t \rightarrow \infty} \sup \left(\|\theta(t)\|+\left\|e\left(t, t_{0}, e_{0}\right)\right\|\right) \\
& \quad \leq \frac{2\|F\| K}{|\sigma(F)|} e^{\frac{4 L}{\sigma(F) \mid}} .
\end{aligned}
$$

The proof is thus complete.
Corollary 3.1. If $f(t, x) \equiv f(x)$, Assumptions 2.12.3 hold, and a control gain matrix $F$ can be chosen such that

$$
\begin{aligned}
& \max _{z \in D}\left\{\lambda_{\max }\left(f_{z}(z)+f_{z}^{T}(z)\right)\right\} \\
&<\lambda_{\min }\left((F H)^{T}+(F H)\right),
\end{aligned}
$$

then, the noise-free synchronization scheme (1)-(3) is exponentially synchronizing and the actual synchronization scheme (1) with (7)-(8) is uniformly synchronizing with an error bound $(2\|F\| K /|\sigma(F)|) e^{\frac{4 L}{\sigma(F) \mid}}$, where

$$
\begin{aligned}
\sigma(F)= & \frac{1}{2}\left\{\max _{z \in D}\left\{\lambda_{\max }\left(f_{z}(z)+f_{z}^{T}(z)\right)\right\}\right. \\
& \left.-\lambda_{\min }\left((F H)^{T}+(F H)\right)\right\} .
\end{aligned}
$$

Proof. Obviously, for any $z \in D, f_{z}(z)+f_{z}^{T}(z)$ is a symmetric matrix. Hence, there exists an orthogonal matrix $P(z)$ such that

$$
\begin{aligned}
\operatorname{diag} & \left\{\lambda_{1}(z), \ldots, \lambda_{n}(z)\right\} \\
& =P(z)\left(f_{z}(z)+f_{z}^{T}(z)\right) P^{T}(z),
\end{aligned}
$$

where $\lambda_{i}(z)$ is an eigenvalue of $f_{z}(z)+f_{z}^{T}(z), i=$ $1,2, \ldots, n$. Also, for $i=1,2, \ldots, n, \lambda_{i}(z)$ is a continuous function of the entries in matrix $f_{z}(z)+f_{z}^{T}(z)$. Hence, $\max _{1 \leq i \leq n}\left\{\lambda_{i}(z)\right\}$ must exist in the bounded and closed set $D$.

From the properties of a symmetric matrix, one has

$$
\begin{aligned}
\lambda_{\max }\{ & \left.(-F H)^{T}+(-F H)+f_{z}(z)+f_{z}(z)^{T}\right\} \\
\leq & \lambda_{\max }\left((-F H)^{T}+(-F H)\right) \\
& +\lambda_{\max }\left(f_{z}(z)+f_{z}(z)^{T}\right) \\
= & -\lambda_{\min }\left((F H)^{T}+(F H)\right) \\
& +\lambda_{\max }\left(f_{z}(z)+f_{z}(z)^{T}\right) \\
\leq & -\lambda_{\min }\left((F H)^{T}+(F H)\right) \\
& +\max _{z \in D}\left\{\lambda_{\max }\left(f_{z}(z)+f_{z}(z)^{T}\right)\right\} .
\end{aligned}
$$


Set $\sigma(F)$ as in (43). Then, it is easy to see that all the conditions of Theorem 3.2 hold and thus the corollary follows. The proof is complete.

Corollary 3.2. If the controller matrix is designed as $F=\mu H^{-1}$, where the constant $\mu$ satisfies

$$
\mu>\frac{1}{2} \lambda_{\max }\left(f_{z}(z)+f_{z}^{T}(z)\right),
$$

then the synchronization error has the following bound:

$$
\|r(t)\| \leq \frac{2 \mu\left\|H^{-1}\right\| K}{\mu-\beta} e^{\frac{4 L}{\mu-\beta}}
$$

where $\beta=(1 / 2) \max _{z \in D}\left[\lambda_{\max }\left(f_{z}(z)+f_{z}^{T}(z)\right)\right]$.

Proof. It is a direct consequence of Theorem 3.2.

\section{Remark 3.2}

(i) In Theorems 3.1-3.2, two kinds of synchronization error estimation formulae have been proposed under which the uniform synchronization with an error bound can be achieved. The synchronization error estimation formula obtained in Theorem 3.1 is derived by using the Lyapunov function method, while the error estimation formulae in Theorem 3.2 and Corollaries 3.1-3.2 are obtained by using the variation of parameters. Although it is one kind of simple estimation, the results in Theorem 3.1 may be relatively conservative due to many inequalities and Lemma 2.3. The example in Sec. 4 will demonstrate the conservative.

(ii) In Theorem 3.1, there is less restriction on function $f$, while in Theorem 3.2, $f$ is required to be differentiable. But, by using Theorem 3.2 and Corollaries 3.1-3.2, the feedback control gain can be easily designed to achieve uniform synchronization with a smaller error bound. For example, by Corollary 3.2, one can choose the controller matrix as $F=\mu H^{-1}$, with $\mu$ satisfying (41), so under the condition of unknown but bounded channel noise, one has

$$
\|r(t)\| \rightarrow 0, \quad \text { as }\left\|H^{-1}\right\| \rightarrow 0 .
$$

In other words, the above-designed feedback controller can achieve uniform synchronization with a smaller error bound subject to unknown but bounded channel noise.

\section{Examples and Numerical Simulations}

In this section, two representative examples are given for illustration. Here, the numerical simulation procedure is coded and executed by using Matlab.

Example 4.1 [Chua's circuit]. Consider the chaotic Chua's circuit:

$$
\left\{\begin{array}{l}
\dot{x}_{1}=\alpha\left(x_{2}-h\left(x_{1}\right)\right), \\
\dot{x}_{2}=x_{1}-x_{2}+x_{3}, \\
\dot{x}_{3}=-\beta x_{2}
\end{array}\right.
$$

where $h\left(x_{1}\right)=m_{1} x_{1}+(1 / 2)\left(m_{0}-m_{1}\right)\left(\left|x_{1}+c\right|-\right.$ $\left.\left|x_{1}-c\right|\right)$.

It is well known that with parameters $m_{0}=$ $-1 / 7, m_{1}=2 / 7, \alpha=9, \beta=14.28, c=1$, for which the sector condition is $[0,1]$, i.e. with slope $\kappa=1$, the circuit generates a double-scroll attractor.

Chua's circuit can be represented in a general Lur'e form: $\dot{\xi}=f(\xi)=A \xi+B \sigma(C \xi)$, with

$$
\begin{aligned}
& \xi=\left(\begin{array}{l}
x_{1} \\
x_{2} \\
x_{3}
\end{array}\right), \quad A=\left(\begin{array}{ccc}
-\alpha m_{1} & \alpha & 0 \\
1 & -1 & 1 \\
0 & -\beta & 0
\end{array}\right), \\
& B=\left(\begin{array}{ccc}
-\alpha\left(m_{0}-m_{1}\right) & 0 & 0 \\
0 & 0 & 0 \\
0 & 0 & 0
\end{array}\right), \quad C=\left(\begin{array}{lll}
1 & 0 & 0 \\
0 & 0 & 0 \\
0 & 0 & 0
\end{array}\right) \text {, } \\
& \sigma(C \xi)=\left(\begin{array}{lll}
\frac{1}{2}\left(\left|x_{1}+c\right|-\left|x_{1}-c\right|\right) & 0 & 0
\end{array}\right)^{T} .
\end{aligned}
$$

For any $\xi, \eta \in D$, one has

$$
\begin{aligned}
\|f(\xi)-f(\eta)\| & =\|A(\xi-\eta)+B(\sigma(C \xi)-\sigma(C \eta))\| \\
& \leq\|A\|\|\xi-\eta\|+\|B\| \kappa\|C(\xi-\eta)\| \\
& =(\|A\|+\kappa\|B\|\|C\|)\|\xi-\eta\| .
\end{aligned}
$$

Here, the Lipschitz constant $L=\|A\|+\kappa\|B\|\|C\|=$ 20.8277.

Set $K=0.1, H=-I$. Choosing $P=I$, $F=-\mu I, \mu>0$, gives

$$
\begin{aligned}
r^{T} g= & \frac{1}{2} r^{T}\left(A+A^{T}+F^{T}+F\right) r \\
& +r^{T} B[\sigma(C(r+y))-\sigma(C y)] \\
\leq & \frac{1}{2} r^{T}\left(A+A^{T}-2 \mu I\right) r+\kappa\|B\|\|C\| r^{T} r \\
= & (12.1727-\mu) r^{T} r \triangleq-p(F, I) r^{T} r .
\end{aligned}
$$


Hence, by Theorem 3.1, one obtains an estimation of the synchronization error as

$$
\|r(t)\| \leq \frac{\|F\| K}{p(F, I)-2 L}=\frac{\mu}{\mu-53.8281} \cdot 10^{-1} .
$$

Obviously, the function $\varphi(\mu)=(\mu /(\mu-53.8281))$. $10^{-1}$ is strictly decreasing for $\mu>53.8281$ and $\lim _{\mu \rightarrow \infty} \varphi(\mu)=10^{-1}$. Therefore, by setting $\mu \gg$ 53.8281 to be sufficiently large, the synchronization error will be less than 0.1 .

In simulations, initial values are $\left[\begin{array}{lll}x_{1} & x_{2} & x_{3}\end{array}\right.$ $\left.\begin{array}{lll}y_{1} & y_{2} & y_{3}\end{array}\right]=\left[\begin{array}{llllll}0.2 & 0.3 & 0.1 & 1.2 & 1.3 & 1.1\end{array}\right], \mu=$ $100, K=0.1$. Some noise is added. The noise is uniformly distributed on $[-K, K]$, generated by using $(\operatorname{rand}(1)-0.5) \times 2 \times K$ in Matlab. The errors of synchronization are shown in Fig. 2, from which one can observe that all the synchronization errors are less than 0.15 , somewhat better than 0.2166 estimated by $(51)$.

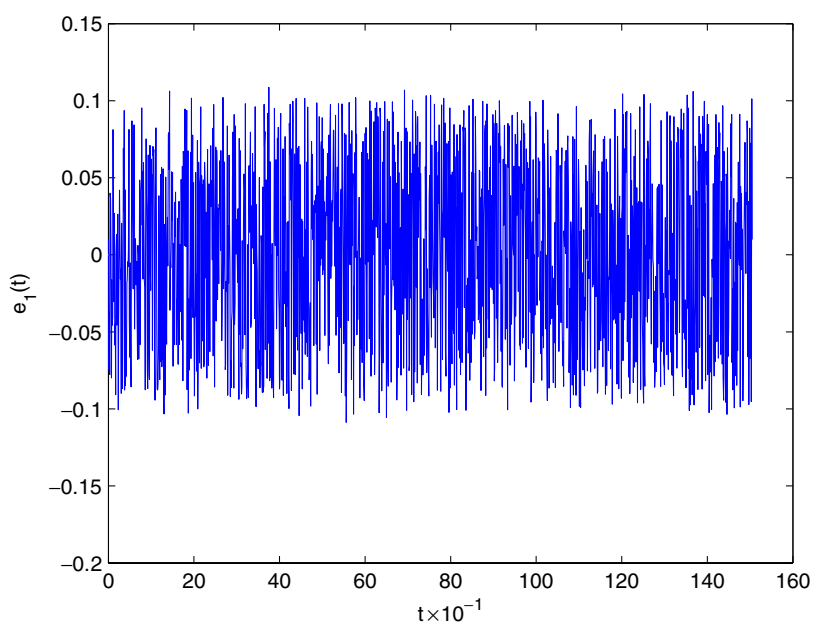

Example 4.2 [Colpitts' Oscillator]. Consider Colpitts' oscillator:

$$
\left\{\begin{array}{l}
\dot{x}_{1}=\alpha x_{2} \\
\dot{x}_{2}=-\sigma\left(x_{1}+\gamma x_{2}+x_{3}\right) \\
\dot{x}_{3}=\beta\left(x_{2}+a_{1} x_{1}+a_{3} x_{1}^{3}\right)
\end{array}\right.
$$

where $\alpha, \beta, \sigma, a_{1}, \gamma, a_{3} \in R$. With parameters $\alpha=$ $2.4, \beta=2.2, \sigma=1, \gamma=0.252, a_{1}=1$, and $a_{3}=-0.2$, the system is chaotic.

Letting $X=\left(x_{1}, x_{2}, x_{3}\right)^{T}$ convert Colpitts' oscillator to

$$
\dot{X}=A X+\Phi(X)
$$

where

$A=\left(\begin{array}{ccc}0 & \alpha & 0 \\ -\sigma & -\gamma \sigma & -\sigma \\ a_{1} \beta & \beta & 0\end{array}\right)$ and $\Phi(X)=\left(\begin{array}{c}0 \\ 0 \\ a_{3} \beta x_{1}^{3}\end{array}\right)$.

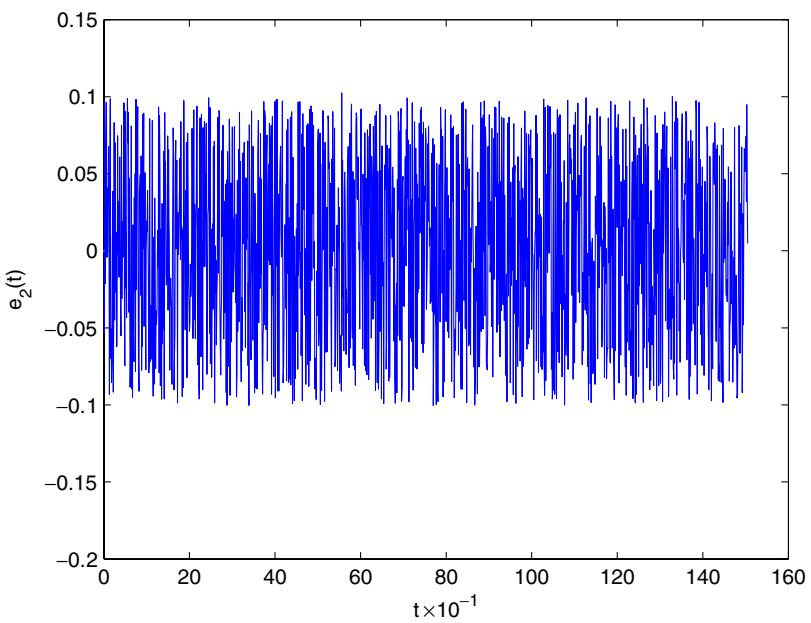

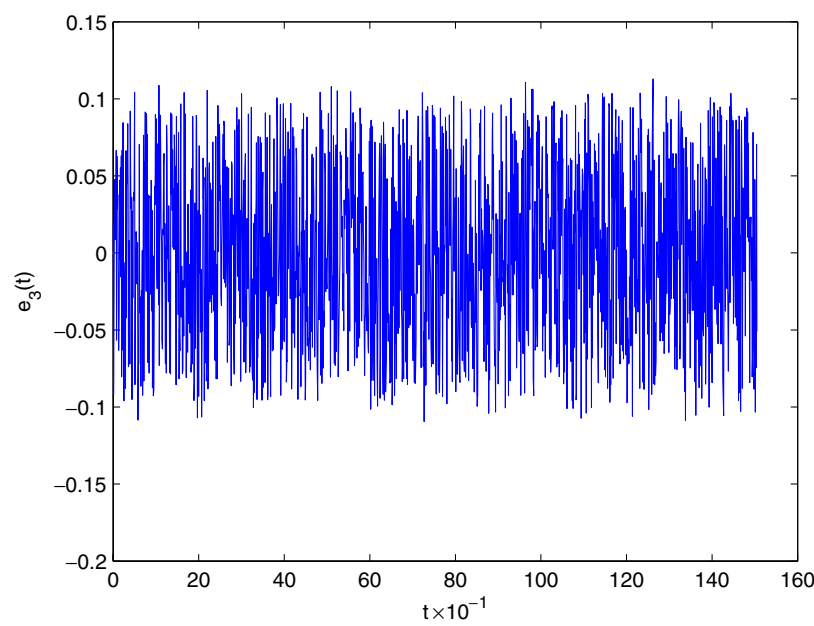

Fig. 2. Synchronization errors $e_{1}-e_{2}-e_{3}$ under $H=-I, F=-100 I$, and $K=0.1$. 

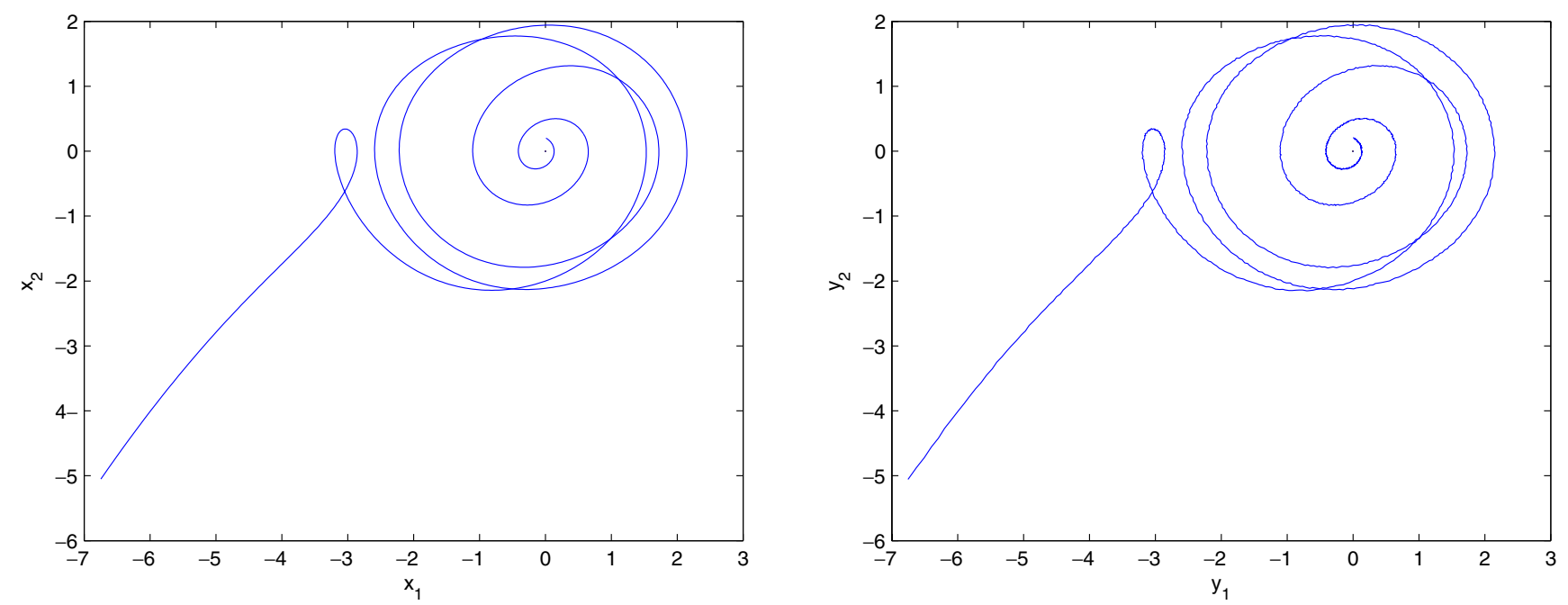

Fig. 3. Attractors of $x_{1}-x_{2}$ (left) and $y_{1}-y_{2}$ with $H=10 I, F=10 I$, and $K=0.1$ (right).
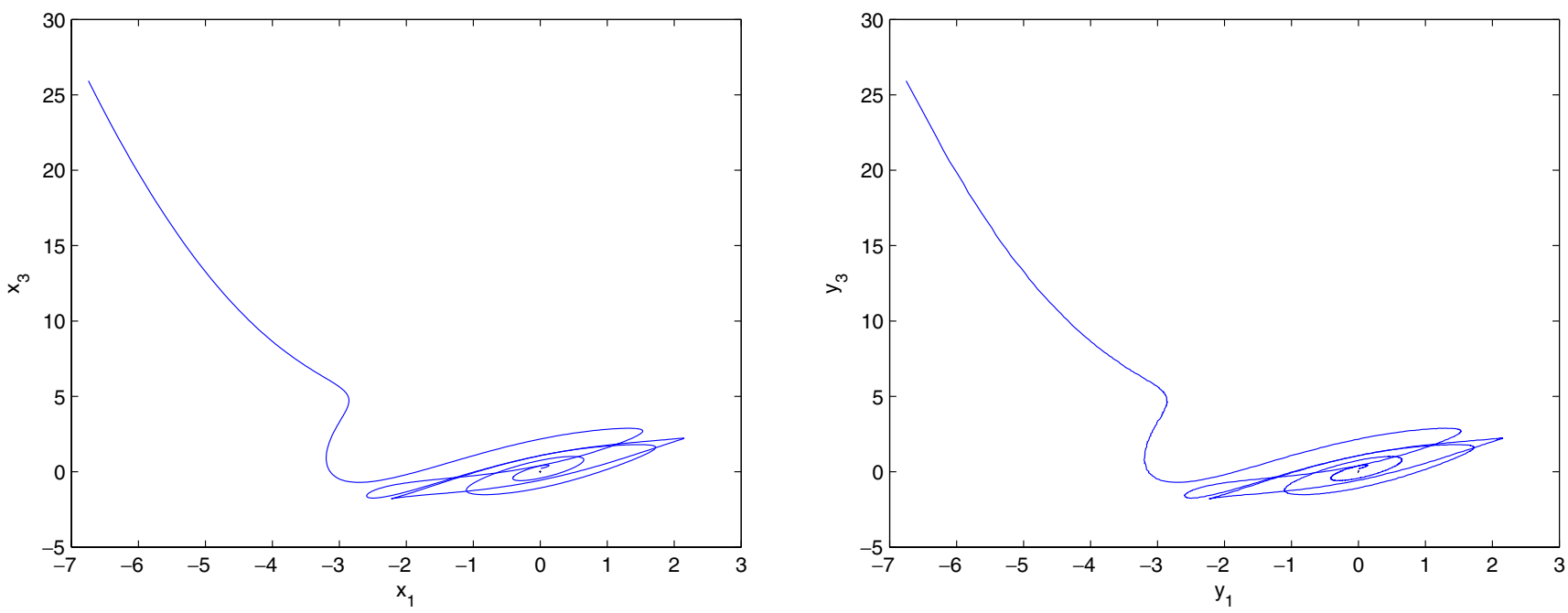

Fig. 4. Attractors of $x_{1}-x_{3}$ (left) and $y_{1}-y_{3}$ with $H=10 I, F=10 I$, and $K=0.1$ (right).
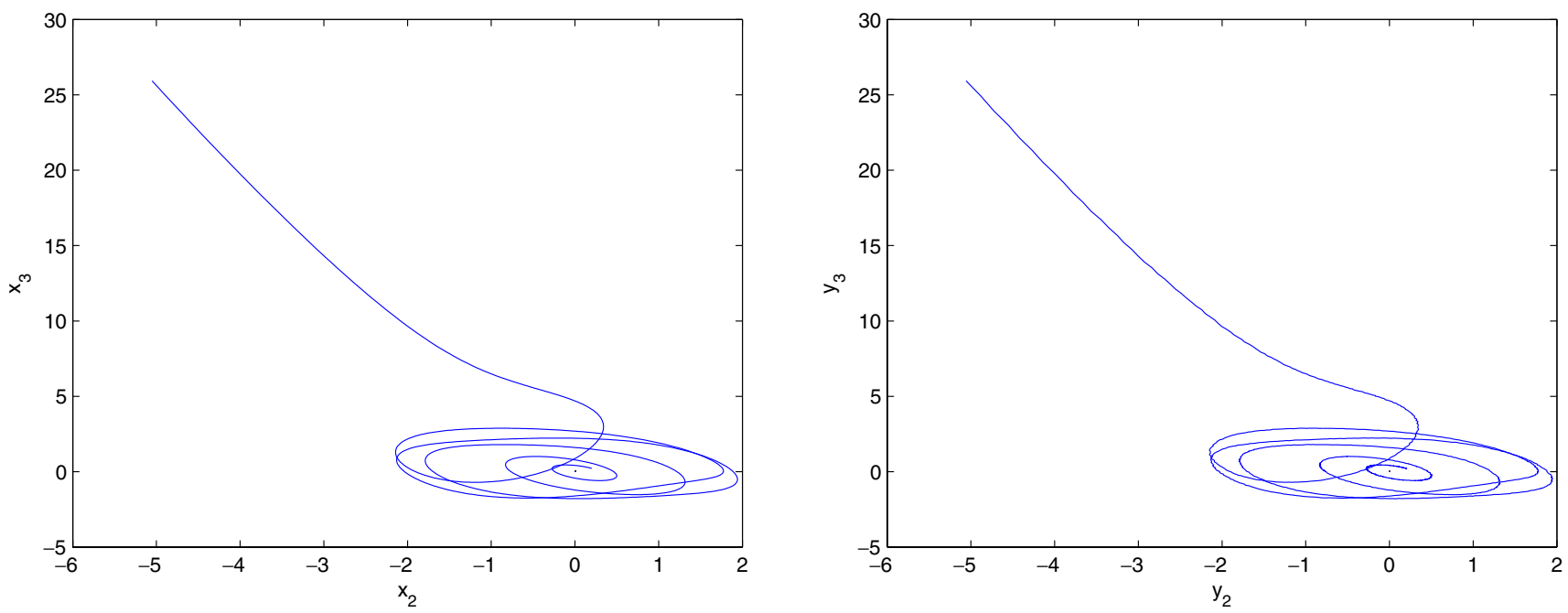

Fig. 5. Attractors of $x_{2}-x_{3}$ (left) and $y_{2}-y_{3}$ with $H=10 I, F=10 I$, and $K=0.1$ (right). 

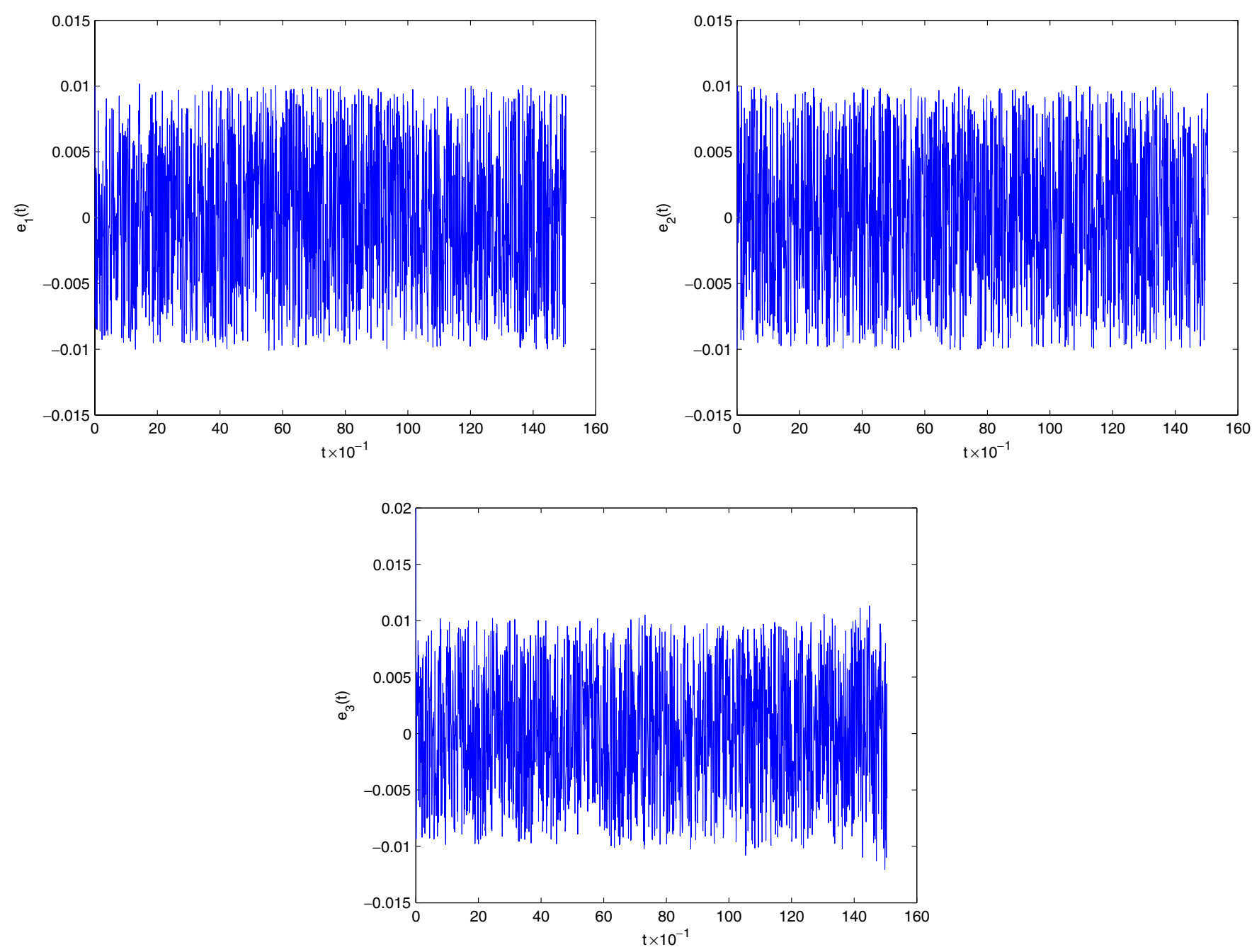

Fig. 6. Synchronization errors $e_{1}-e_{2}-e_{3}$ under $H=10 I, F=10 I$, and $K=0.1$.

For any $X, Y \in D$, one has

$$
\begin{aligned}
& \|f(X)-f(Y)\|=\|A(X-Y)+\Phi(X)-\Phi(Y)\| \\
& \quad \leq\|A\|\|X-Y\|+6 \delta^{2}\left|a_{3} \beta\right|\|X-Y\| \\
& \quad=\left(\|A\|+6 \delta^{2}\left|a_{3} \beta\right|\right)\|X-Y\| .
\end{aligned}
$$

Here, the Lipschitz constant $L=\|A\|+6 \delta^{2}\left|a_{3} \beta\right|=$ $3.7322+2.64 \delta^{2}$, and $\|X\|,\|Y\| \leq \delta$.

Note that,

$$
\begin{aligned}
& \lambda_{\max }\left(f_{X}(X)+f_{X}^{T}(X)\right) \\
& \quad=\lambda_{\max }\left\{A+A^{T}+\left(\begin{array}{ccc}
0 & 0 & 3 a_{3} \beta x_{1}^{2} \\
0 & 0 & 0 \\
3 a_{3} \beta x_{1}^{2} & 0 & 0
\end{array}\right)\right\} \\
& \leq \lambda_{\max }\left(A+A^{T}\right)+\left|3 a_{3} \beta x_{1}^{2}\right| \\
& \leq 14.8636+1.32 \delta^{2} .
\end{aligned}
$$

Since $H$ is nonsingular by assumption, one may let $F=\mu H^{-1}$, with $\mu$ satisfying

$$
\mu>\frac{1}{2}\left(14.8636+1.32 \delta^{2}\right)
$$

Then, let $\sigma(F)=-\left(\mu-7.4318-0.66 \delta^{2}\right)$. By Corollary 3.1 , the synchronization error satisfies

$$
\begin{aligned}
\|r(t)\| & \leq \frac{2\|F\| K}{|\sigma(F)|} e^{\frac{4 L}{|\sigma(F)|}} \\
& =\frac{2 \mu K\left\|H^{-1}\right\|}{\mu-7.4318-0.66 \delta^{2}} e^{\frac{4\left(3.7322+2.64 \delta^{2}\right)}{\mu-7.4318-0.66 \delta^{2}}} .
\end{aligned}
$$

Moreover, the function

$$
\varphi(\mu)=\frac{2 \mu K\left\|H^{-1}\right\|}{\mu-7.4318-0.66 \delta^{2}} e^{\frac{4\left(3.7322+2.64 \delta^{2}\right)}{\mu-7.4318-0.66 \delta^{2}}}
$$

reaches its maximum value at $\mu^{*}=(7.4318+$ $\left.0.66 \delta^{2}\right)^{2} /\left(22.36+11.22 \delta^{2}\right)$ and it is strictly 

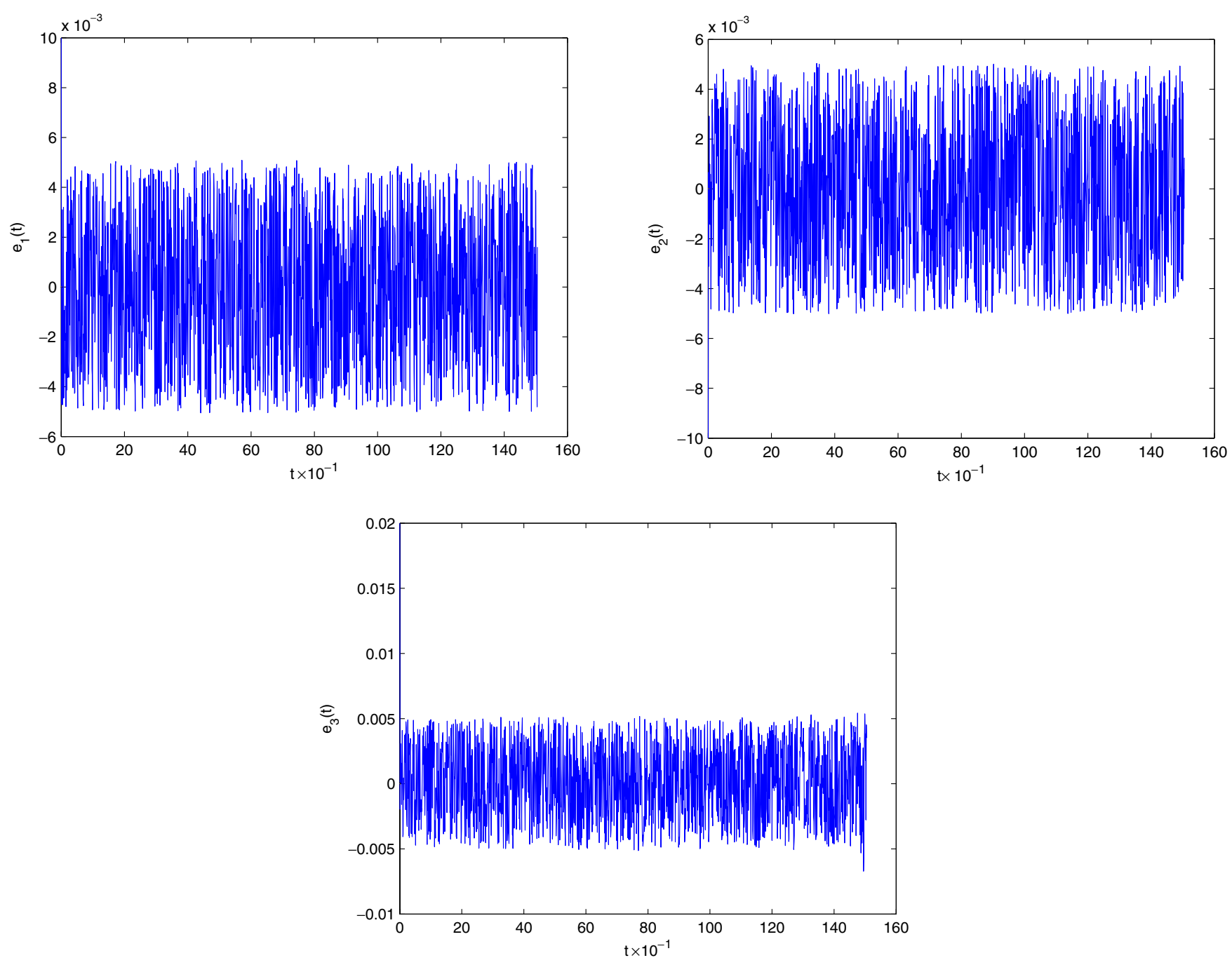

Fig. 7. Synchronization errors $e_{1}-e_{2}-e_{3}$ under $H=20 I, F=5 I$, and $K=0.1$.

decreasing for $\mu>\mu^{*}$ and $\lim _{\mu \rightarrow \infty} \varphi(\mu)=$ $2 K\left\|H^{-1}\right\|$. Therefore, by setting $\mu>\max \left\{\mu^{*}\right.$, $\left.7.4318+0.66 \delta^{2}\right\}$, the synchronization error will be less than $2 K\left\|H^{-1}\right\|$.

Here, let $\mu \gg \max \left\{\mu^{*}, 7.4318+0.66 \delta^{2}\right\}$ and $\left\|H^{-1}\right\|$ be sufficiently small. Then, the controller matrix $F=\mu H^{-1}$ achieves uniform synchronization within an error bound given above.

In simulations, initial values are $\left[\begin{array}{lll}x_{1} & x_{2} & x_{3}\end{array}\right.$ $\left.\begin{array}{lll}y_{1} & y_{2} & y_{3}\end{array}\right]=\left[\begin{array}{llllll}0.2 & 0.3 & 0.1 & 1.2 & 1.3 & 1.1\end{array}\right], \mu=$ $100, K=0.1, \delta=4$. Some noise is added. The noise is uniformly distributed on $[-K, K]$, generated by using $(\operatorname{rand}(1)-0.5) \times 2 \times K$ in Matlab.

First, let $H=10 I$, i.e. $F=10 I$. The attractors of the master and slave systems and the errors of synchronization are shown in Figs. 4 and 5, respectively, from which one can observe that all the synchronization errors are less than 0.015 , consistent with the results obtained above $\left(<2 K\left\|H^{-1}\right\|=\right.$ 0.02) based on Corollary 3.2 .

To achieve uniform synchronization with an error bound $\epsilon=0.01$, one can set $H=20 I$, i.e. $F=5 I$. The attractors of the master and slave systems and the errors of synchronization are shown in Figs. 3-7, respectively, from which one can observe that the designed controller achieves the expected uniform synchronization with the required error bound $\epsilon=0.01$.

Remark 4.1. Examples 4.1-4.2 validate and demonstrate the theoretical results obtained in this paper. Meanwhile, one can see that the theoretical results (especially in Example 4.1) are relatively conservative. But, from Example 4.2, one can see that the estimation of synchronization errors indeed helps the design of the feedback controller to achieve 
uniform synchronization within a given error bound.

\section{Conclusions}

In this paper, exponential and uniform synchronizations as well as synchronization error bounds have been investigated for the general master-slave chaotic synchronization scheme via feedback control, possibly subject to unknown but bounded noise disturbances. By employing the methods of Lyapunov functions and nonlinear variation of parameters, some estimation formulae of the synchronization error bounds have been derived. The estimation formulae can be applied to the design of the feedback controllers, which is used to force the master-slave chaotic systems to synchronize up to a relatively small error bound, even in the case with unknown but bounded noise disturbances.

It is well known that a chaotic system has complex dynamical behaviors and possesses some special features that make chaotic synchronization useful for secure communications. It is also well known, however, that chaotic synchronization is often destroyed by channel noise. In fact, it is generally difficult to achieve synchronization if there exists noise in the signal transmission channel. Therefore, it is essential to estimate the synchronization errors. The estimation formulae of synchronization error bounds obtained in this paper will help in the design of a feedback controller to achieve uniform synchronization within an arbitrarily given small error bound subject to unknown but bounded channel noise.

Two examples, the chaotic Chua's circuit and Colpitts' oscillator, have been simulated, validating and also demonstrating the effectiveness of the theoretical results of the paper.

\section{References}

Brauer, F. [1966] "Perturbations of nonlinear systems of differential equations," J. Math. Anal. Appl. 14, 198206.

Carroll, T. L. \& Pecora, L. M. [1991] "Synchronization in chaotic circuits," IEEE Trans. Circuts Syst.-I 38, $453-456$.

Chen, G. \& Lai, D. [1996] "Feedback control of Lyapunov exponents for discrete-time dynamical systems," Int. J. Bifurcation and Chaos 6, 1341-1349.

Chen, G. \& Dong, X. [1998] From Chaos to Order: Methodologies, Perspectives and Applications (World Scientific, Singapore).
Chen, G. [2000] Controlling Chaos and Bifurcations in Engineering Systems (CRC Press, Boca Raton, FL).

Curran, P. F. \& Chua, L. O. [1997] "Absolute stability theory and the synchronization problem," Int. J. Bifurcation and Chaos 7, 1375-1382.

Fang, J. Q. \& Hong, Y. [1999] "Switching manifold approach to chaos synchronization," Phys. Rev. E 59, 2523-2526.

Grassi, G. \& Mascolo, S. [1997] "Nonlinear observer design to synchronize hyperchaotic systems vis a scalar signal," IEEE Trans. Circuts Syst.-I 44, 10111014.

Jiang, G. P., Tang, W. K. S. \& Chen, G. [2006] "A state-observer-based approach for synchronization in complex dynamical networks," IEEE Trans. Circuits Syst.-I 53, 2739-2745.

Kocarev, L. \& Parlitz, U. [1995] "General approach for chaotic synchronization with application to communication," Phys. Rev. Lett. 74, 5028-5031.

Kocarev, L., Maggio, G. M., Ogorzalek, M., Pecora, L. \& Yao, K. [2001] "Introduction to the special issue," IEEE Trans. Circuts Syst.-I 48, 1385-1386.

Li, C. \& Chen, G. [2004a] "Synchronization in general complex dynamical networks with coupling delays," Phys. A 343, 263-278.

Li, Z. \& Chen, G. [2004b] "Robust adaptive synchronization of uncertain dynamical networks," Phys. Lett. A 324, 166-178.

Liu, B., Liu, X., Chen, G. \& Wang, H. [2005] "Robust impulsive synchronization of uncertain dynamical networks," IEEE Trans. Circuts Syst.-I 52, 1431-1441.

Liu, B., Teo, K. L. \& Liu, X. [2007] "Global synchronization of dynamical networks with coupling timedelays," Phys. Lett. A 368, 53-63.

Liu, B. \& Marquez, H. J. [2008] "Uniform stability of discrete delay systems and synchronization of discrete delay dynamical networks via Razumikhin technique," IEEE Trans. Circuits Syst.-I 55(11).

Lü, J. \& Chen, G. [2005] "A time-varying complex dynamical model and its controlled synchronization criteria," IEEE Trans. Autom. Contr. 50, 841846.

Pecora, L. M. \& Carroll, T. L. [1990] "Synchronization in chaotic systems," Phys. Rev. Lett. 64, 821-824.

Pecora, L. M., Carroll, T. L., Johnson, G., Mar, D. \& Fink, K. S. [2000] "Synchronization stability in coupled oscillator arrays: Solution for arbitrary configurations," Int. J. Bifurcation and Chaos 10, 273-290.

Rodrigues, H. M., Alberto, L. F. C. \& Bretas, N. G. [2000] "On the invariance principle: Generalizations and applications to synchronization," IEEE Trans. Circuits Syst.-I 47, 730-738.

Suykens, J. A. K., Curran, P. F., Vandewalle, J. \& Chua, L. O. [1997] "Robust nonlinear $H_{\infty}$ synchronization of chaotic Lur'e systems," IEEE Trans. Circuts Syst.-I 44, 891-904. 
Suykens, J. A. K., Curran, P. F., Vandewalle, J. \& Chua, L. O. [1999] "Robust synthesis for master-slave synchronization of Lur's systems," IEEE Trans. Circuts Syst.-I 46, 841-850.

Teel, A. R. [1998] "Connections between Razumikhintype theorems and the ISS nonlinear small gain theorem," IEEE Trans. Autom. Contr. 43, 960-964.

Wang, X. \& Chen, G. [2002] "Synchronization in scalefree dynamical networks: Robustness and fragility," IEEE Trans. Circuts Syst.-I 49, 54-62.

Wu, C. W. \& Chua, L. O. [1994] "A unified framework for synchronization and control of dynamical systems," Int. J. Bifurcation and Chaos 4, 979-989.

Wu, C. W., Yang, T. \& Chua, L. O. [1996] "On adaptive synchronization and control of nonlinear dynamical systems," Int. J. Bifurcation and Chaos $\mathbf{6}$, 455-471.
Wu, C. W. [2003] "Perturbation of coupling matrices and its effect on the synchronizability in arrays of coupled chaotic systems," Phys. Lett. A 319, 495-503.

Xiang, J. \& Chen, G. [2007] "On the V-stability of complex dynamical networks," Automatica 43, 10491057.

Yalcin, M. E., Suykens, J. A. K. \& Vandewalle, J. [2001] "Master-slave synchronization of Lur'e systems with time-delay," Int. J. Bifurcation and Chaos 11, 17071772.

Yang, T. \& Chua, L. O. [1997] "Impulsive stabilization for control and synchronization of chaotic systems: Theory and application to secure communication," IEEE Trans. Circuts Syst.-I 44, 976-988.

Yang, T. [2001] Impulsive Control Theory (SpringerVerlag). 